\title{
MODELAGEM CFD DE UM REATOR RISER-FCC USANDO O MODELO CINETICO DE LUMPING CONTINUO
}

\author{
J.L. GÓMEZ ${ }^{1 *}$, W. P. MARTIGNONI ${ }^{2}$, R. OCONE ${ }^{3}$, M. MORI $^{1}$ \\ ${ }^{1}$ Universidade Estadual de Campinas, Faculdade de Engenharia Química \\ ${ }^{2}$ PETROBRAS, AB-RE/TR/OT \\ ${ }^{3}$ Heriot-Watt University, Department of Chemical Engineering \\ "e-mail: jogover@gmail.com
}

\begin{abstract}
RESUMO
Sabe-se que os efeitos da hidrodinâmica sobre a seletividade e conversão dos produtos devem ser considerados na descrição cinética das reações do craqueamento catalítico fluidizado (FCC). Embora, os testes experimentais sejam bastante desejados, seu custo e tempo muitas vezes os tornam inviáveis, especialmente em equipamentos de escala industrial. Desse modo, a fluidodinâmica computacional (CFD) considera-se uma ferramenta útil no projeto e otimização desses processos. Não obstante, a modelagem das reações FCC não é uma tarefa fácil, já que, envolve um alto número de reações e componentes, por isso frequentemente tem-se utilizado abordagens cinéticas baseadas em pseudo-componentes para representar as reações, porém existem algumas limitações na descrição precisa destes pseudo-componentes. Neste trabalho propõe-se a utilização de uma abordagem inovadora em que funções de distribuição da espécie química são aplicadas para descrever a cinética das reações FCC sendo considerada a mistura reativa como continua. No estudo pretende-se avaliar a implementação do modelo cinético de lumping contínuo em junção com a hidrodinâmica associada de um leito fluidizado circulante (CFB) para predizer o rendimento dos produtos de uma unidade FCC industrial. Resultados preliminares mostraram uma adequada concordância com dados experimentais reportados na literatura, sendo possível desse modo, descrever reações em misturas complexas com um número reduzido de parâmetros cinéticos além de maior detalhamento das reações de craqueamento.
\end{abstract}

\section{INTRODUÇÃO}

Os leitos fluidizados são utilizados para diversos processos na indústria química, especialmente em processos com reações químicas, como o craqueamento catalítico fluidizado. O objetivo dos processos de FCC é aumentar a relação $\mathrm{H} / \mathrm{C}$ convertendo hidrocarbonetos de alto peso molecular em produtos de maior valor agregado tais como a produção de gasolina e diesel. Desse modo, as unidades de FCC oferecem uns dos maiores potenciais no aumento da rentabilidade das refinarias sendo segundo alguns autores como o coração da indústria do refino (FAHIM, ALSAHHAF e ELKILANI, 2010).

Em vista que os processos FCC envolvem sistemas reativos em escoamentos multifásicos com múltiplos componentes, os efeitos hidrodinâmicos sobre a seletividade e conversão são importantes na cinética das reações e, por conseguinte uma das maiores preocupações na sua modelagem trata-se em descrever cuidadosamente a química das mesmas. (PÄRSSINEN e ZHU, 2001). 


\subsection{Descrição do Processo}

De acordo com Gray e Handwerk (2001) a unidade FCC compõe-se de três seções principalmente: reação, regeneração e separação. A seção de reação consiste em um reator ascendente (riser), em que a carga do processo (gasóleo) é introduzida em conjunto com uma mistura de catalisador e vapor de água como mostra a Figura 1. A carga é alimentada no riser através de injetores, cujo objetivo, é dispersar finamente em gotículas a carga para favorecer sua vaporização. Entretanto, o catalisador além da sua atividade catalítica, serve como transporte do calor necessário na vaporização da carga (650 $-710^{\circ} \mathrm{C}$ ), assim como fornecer a energia térmica para que ocorram as reações de craqueamento (SADEGHBEIGI, 2000).

Figura 1 - Esquema de uma Unidade FCC

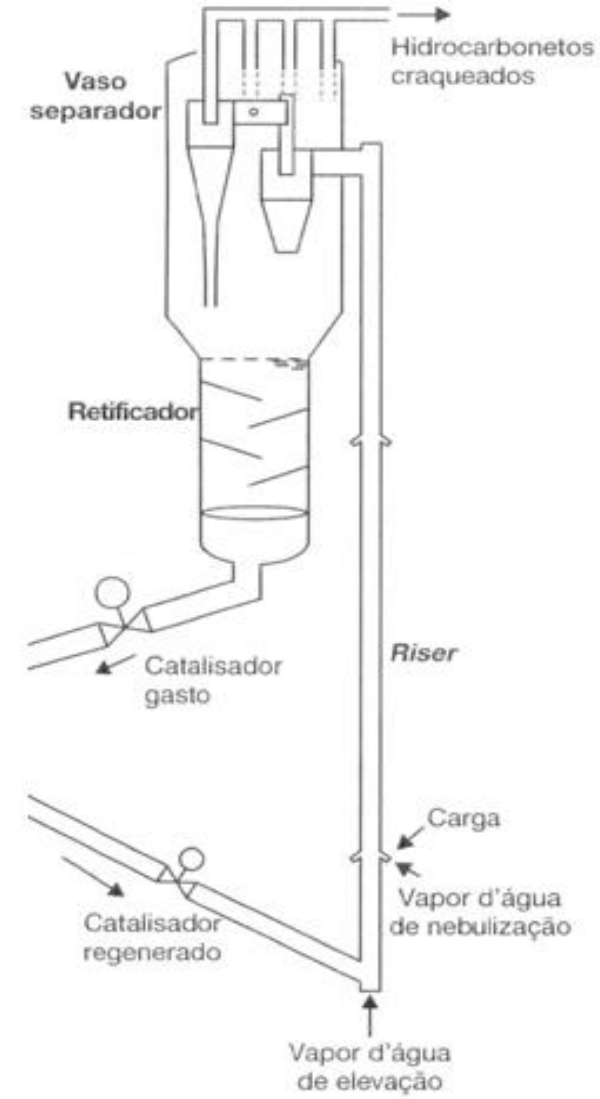

Fonte: Brasil et al. (2012).
A fluidização do sistema é feita devido ás grandes vazões de vapor de água e hidrocarbonetos vaporizados, realizando o arraste das partículas sólidas do catalisador em direção ao topo do riser em altas velocidades. Nesse percurso, as moléculas de maior peso são quebradas em moléculas mais leves ao contato com o catalisador, sob condições de pressões moderadas (1-5 bar) e altas temperaturas $\left(480-550{ }^{\circ} \mathrm{C}\right)$ conforme o tipo de carga, catalisador ou produtos de interesse (FAHIM, ALSAHHAF e ELKILANI, 2010).

Finalmente, após passar pelo riser, o catalisador é separado dos produtos das reações de forma rápida na seção de separação (stripper) e finalmente o coque depositado no catalisador é queimado na seção de regeneração para recuperar sua atividade catalítica.

\subsection{Reações de craqueamento catalítico}

Visto que nas reações de craqueamento catalítico do processo FCC, os diversos componentes (e.g., parafinas, naftênicos, olefinas, aromáticos, poli aromáticos) que compõem a carga reagem de formas diferentes; é importante considerar um esquema cinético adequado para obter uma maior compressão dos fenômenos que ocorrem no sistema, bem como as caraterísticas e quantidades dos produtos (BASAK et al., 2004).

Devido ao grande número de espécies químicas, considerar um esquema completo envolvendo todas as reações FCC pode ser bastante complexo, por isso, algumas simplificações são necessárias. Uma dessas simplificações consiste em aglomerar vários componentes químicos com características similares em um componente só, chamado lump. Assim, estes podem ser utilizados para descrever a cinética das reações em termos de um número reduzido de pseudocomponentes (FAHIM, ALSAHHAF e ELKILANI, 2010). 
$\mathrm{Na}$ literatura existem muitos trabalhos que reportam o comportamento dinâmico das reações FCC ao longo do riser (GAO et al., 1999; CHANG et al., 2012) porem, a maioria desses modelos é baseada em abordagens tradicionais de lumps discretos, que podem apresentar desvantagens na capacidade de predição do rendimento dos produtos, quando são mudadas as características da carga do processo (Feedstock) (ANCHYETA, 2011). Outra das limitações dessa abordagem devese ao elevado numero de parâmetros cinéticos necessários conforme aumenta o numero dos pseudo-componentes.

Por conseguinte, tem-se realizado grandes esforços focados na descrição detalhada da cinética envolvendo o maior número de reações químicas possíveis, em que uma série reduzida de parâmetros cinéticos e maiores informações acerca da caracterização do feedstock sejam requeridas, em que o modelo de lumping contínuo apresenta grandes vantagens.

\subsubsection{Modelo cinético lumping contínuo}

Nessa abordagem a mistura reativa é considerada sendo uma distribuição contínua em relação a propriedades ou características que representam os pseudocomponentes da mistura (e.g. número de carbonos, peso molecular, ponto de ebulição TBP, etc.).

Conforme a literatura, nas reações de craqueamento os compostos mais pesados reagem com maior rapidez que aqueles compostos mais leves, por conseguinte, a reatividade de cada componente pode ser associada com uma propriedade intrínseca do através de uma função. Essa função foi representada por Laxminarasimhan, Verna e Ramachandran (1996) na Equação 1 como uma lei de potência, já que maioria de dados reportados na literatura segue esse comportamento.

$$
\frac{k}{k_{\max }}=\theta^{1 / \alpha}
$$

Por outro lado, a concentração de qualquer pseudocomponente (i) da mistura reativa pode ser avaliada da seguinte forma:

$$
C_{i}(t) d i=C(\theta, t) d \theta
$$

Em que, $d \theta$ representa o pseudocomponente definido a partir de uma propriedade ou característica deste e $C(\theta, t)$ corresponde à função de distribuição de concentração. Realizando uma substituição de variáveis $(i \rightarrow k)$ na Equação 2 podemos encontrar a seguinte expressão:

$C(\theta, t) d \theta=C(k, t) D(k) d k$

Onde,

$D(k)=\frac{d i}{d k}=\frac{d i}{d \theta} \frac{d \theta}{d k}$

Entretanto, derivando a Equação $1 \mathrm{e}$ assumindo que $d i / d \theta \approx N$ quando o numero total de pseudocomponente $N \rightarrow \infty$, o operador $D(k)$ torna-se:

$D(k)=N \frac{\alpha}{k_{\max }^{\alpha}} k^{\alpha-1}$

Finalmente, a representação continua da mistura pode ser dada pela seguinte equação:

$C(t)=\int_{0}^{\infty} C(k, t) D(k) d k$

Entretanto, o balanço de material da espécie com reatividade $(k)$ pode ser vista na Figura 2. Esta pode ser descrita para um sistema fechado de acordo com a seguinte equação.

$$
\begin{aligned}
& \frac{d C(k, t)}{d t}=\int_{k}^{K_{\max }} p(k, K) \cdot K \cdot C(K, t) D(K) d K \\
& -k C(k, t)
\end{aligned}
$$


Figura 2 - Esquema de Balanço de material

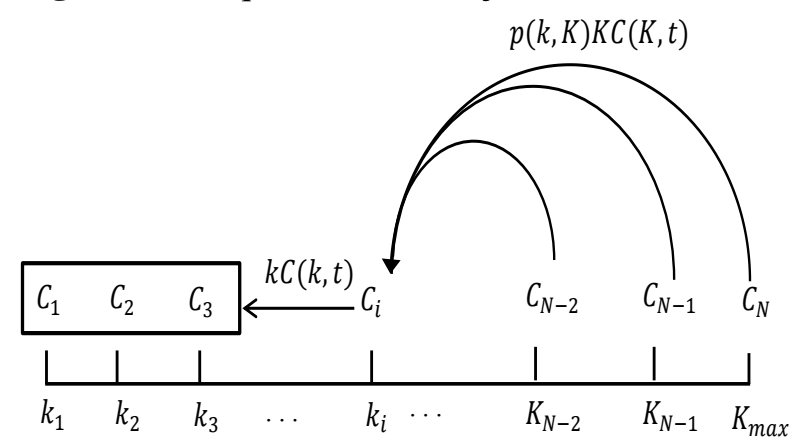

Fonte: Autor.

Em que, $p(k, K)$ determina a formação da espécie com reatividade $(k)$ a partir de espécies com reatividade maior (K) e pode ser representada através de uma função Gaussiana assimétrica da reatividade dos componentes do modelo, de acordo com a as seguintes expressões:

$P(k, K)=\frac{1}{S_{0} \sqrt{2 \pi}}\left[\boldsymbol{e}^{-\left(\frac{\left(\frac{k}{K}\right)^{a_{0}}-0.5}{a_{1}}\right)^{2}}-A-B\right]$

Onde,

$$
\begin{aligned}
& A=e^{-\left(0.5 / a_{1}\right)^{2}} \\
& B=\delta\left[1-\left(\frac{k}{K}\right)\right] \\
& S_{o}=\int_{0}^{K} \frac{1}{\sqrt{2 \pi}}\left[e^{-\left(\frac{\left(\frac{k}{K}\right)^{a_{o}}-0.5}{a_{1}}\right)^{2}}-A-B\right] D(k) d k
\end{aligned}
$$

Os parâmetros kmax, $\alpha, \delta, a 1, a 2$ são utilizados para ajustar o modelo e são específicos do sistema, dependendo do tipo de catalisador, atividade e impurezas presentes na carga. Embora essa abordagem tem-se utilizado para descrever mecanismos reação em misturas complexas em outros processos, tais como a polimerização e hidroconversão (ADAM et al., 2012; ELIZALDE e ANCHEYTA, 2011), ainda não existem trabalhos reportados na literatura em reatores riser-FCC.

\section{MODELAGEM MATEMATICA}

A fim de descrever o escoamento gássólido no reator, foi utilizada uma abordagem Eulerian-Eulerian, em que ambas as fases são consideradas contínuas e interpenetráveis, interagindo entre si. Entretanto o esquema cinético de lumping continuo foi incorporado para descrever as reações.

Nessa abordagem as equações de conservação (movimento, massa e energia) são resolvidas separadamente para cada fase (gás-solido), com termos adicionais que representam as interações entre as mesmas. As equações de transporte são mostradas a seguir:

\subsection{Equações de continuidade}

$$
\frac{\partial\left(\varepsilon_{g} \rho_{g}\right)}{\partial t}+\nabla \cdot\left(\varepsilon_{g} \rho_{g} v_{g}\right)=0
$$

$$
\frac{\partial\left(\varepsilon_{g} \rho_{g}\right)}{\partial t}+\nabla \cdot\left(\varepsilon_{g} \rho_{g} v_{g}\right)=0
$$

\subsection{Equações de movimento}

$$
\begin{aligned}
& \frac{\partial\left(\varepsilon_{g} \rho_{g} v_{g}\right)}{\partial t}+\nabla \cdot\left(\varepsilon_{g} \rho_{g} v_{g} v_{g}\right)=\nabla \cdot \tau_{g}+\varepsilon_{g} \rho_{g} \vec{g} \\
& -\varepsilon_{g} \nabla p-\beta\left(v_{g}-v_{s}\right)
\end{aligned}
$$

$$
\begin{aligned}
& \frac{\partial\left(\varepsilon_{s} \rho_{s} v_{s}\right)}{\partial t}+\nabla \cdot\left(\varepsilon_{s} \rho_{s} v_{s} v_{s}\right)=\nabla \cdot \tau_{s}+\varepsilon_{s} \rho_{s} \vec{g} \\
& +\varepsilon_{s} \nabla p_{s}+\beta\left(v_{g}-v_{s}\right)
\end{aligned}
$$




\subsection{Equações de energia}

$\frac{\partial\left(\varepsilon_{g} \rho_{g} h_{g}\right)}{\partial t}+\nabla \cdot\left(\varepsilon_{g} \rho_{g} h_{g} v_{g}\right)=\nabla \cdot\left[\varepsilon_{g} \lambda_{g} \nabla T\right]$

$-Q_{r}+Q_{g / s}$

$\frac{\partial\left(\varepsilon_{s} \rho_{s} h_{s}\right)}{\partial t}+\nabla \cdot\left(\varepsilon_{s} \rho_{s} h_{s} v_{s}\right)=\nabla \cdot\left[\varepsilon_{s} \lambda_{s} \nabla T\right]$

$-Q_{g / s}$

\subsection{Equações da espécie química}

$\frac{\partial\left(\varepsilon_{g} \rho_{g} Y_{g}^{i}\right)}{\partial}+\nabla \cdot\left(\varepsilon_{g} \rho_{g} Y_{g}^{i} v_{g}\right)=\nabla \cdot\left(\varepsilon_{g} \rho_{g} \Gamma_{i} \nabla Y_{g}^{i}\right)$

$+\left(1-\varepsilon_{g}\right) \phi R_{i}$

Em que, segundo o modelo cinético de lumping contínuo a taxa liquida de reação $(R i)$ é representada da seguinte maneira:

$R_{i}=\int_{k}^{K_{\max }} p(k, K) \cdot K \cdot C(K, t) D(K) d K$

$-k C(k, t)$

\section{MODELO CFD}

No estudo foi considerado um reator riser-FCC industrial com diâmetro $0.71 \mathrm{~m}$ e altura $33 \mathrm{~m}$. Na fase particulada foram usadas propriedades do catalisador FCC com uma relação de 7.2 catalisador/gasóleo e temperatura de $900 \mathrm{~K}$ na entrada do reator. Entretanto, na fase gás foi considerada uma mistura de gasóleo e vapor de agua com velocidade superficial de $9 \mathrm{~m} / \mathrm{s}$ e temperatura de 550K. Neste estudo o software CFD comercial ANSYS CFX 14.0 foi usado para simular o escoamento através do reator.

A reatividade dos pseudocomponentes da mistura foi associada ao peso molecular dos mesmos e os parâmetros do modelo foram estabelecidos a partir de informações da literatura baseada em outros processos similares de craqueamento. Desse modo, foram incorporadas as reações cinéticas através de uma sub-rotina Fortran utilizando o modelo de lumping contínuo. Os pseudocomponentes da mistura reativa foram estabelecidos conforme mostra a Tabela1.

Tabela 1 - Definição dos pseudo-componentes.

\begin{tabular}{ccc}
\hline Índice & Lump & $\begin{array}{c}\text { Peso molecular } \\
\text { médio }\end{array}$ \\
\hline 1 & Coke & - \\
2 & C1-C2 & 25 \\
3 & C3-C4 & 50 \\
4 & C5-C7 & 100 \\
5 & C8-C11 & 150 \\
6 & C12-C14 & 200 \\
7 & C15-C21 & 300 \\
8 & C22-C28 & 400 \\
9 & $>$ C29 & 500 \\
\hline
\end{tabular}

Fonte: Autor.

Figura 3 - Domínio do reator riser-FCC
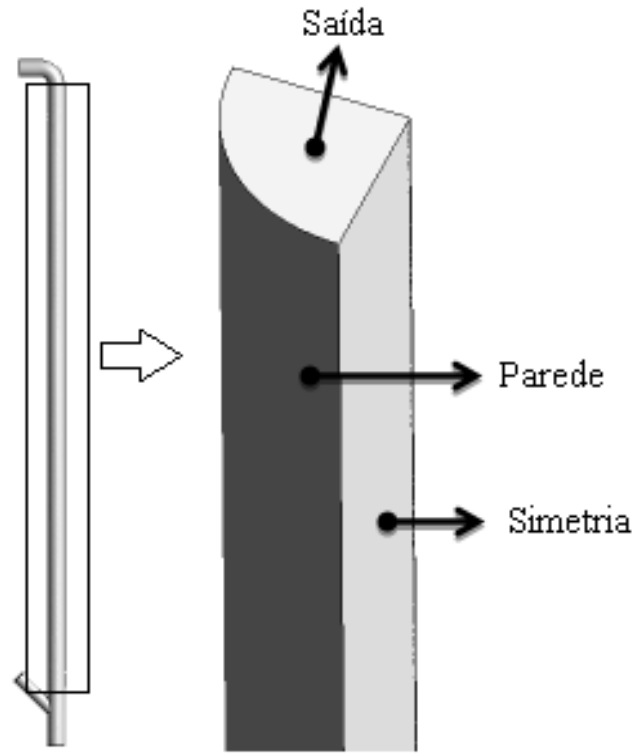

Fonte: Autor.

A fim de caracterizar a turbulência do escoamento foi utilizado na fase gás o modelo de turbulência $k-\varepsilon$ amplamente usado na literatura (WILCOX, 1993). Entretanto, o modelo de arraste de Gidaspow (1994) foi considerado para quantificar as interações 
entre ambas as fases. No intuito de diminuir o esforço computacional, foram utilizadas condições de simetria na geometria em que foi considerada somente $1 / 4$ do domínio do riser como pode ser visto na Figura 3. Por outro lado, condições de parede na fase gás de não escorregamento e escorregamento livre na fase sólido também foram estabelecidas.

Foi gerada uma malha estruturada 3D baseada em informações de malhas feitas em outros trabalhos com dimensões e condições de operação similares as expostas acima. Autores como Li et al. (2013) criaram uma malha com 75.000 elementos para $1 / 4$ da geometria do riser enquanto que Chang et al. (2012) consideraram cerca de 380.000 elementos em uma geometria completa do riser. Desse modo, foram considerados 290.000 elementos levando em conta parâmetros de qualidade de malha e um numero suficientes de elementos para garantir a independências numéricas dos resultados. Além disso, foi feito um refinamento na região da parede como mostra a Figura 4 para predizer da melhor maneira possível essa região já que se esperam gradientes mais elevados.

Figura 4 - Malha computacional

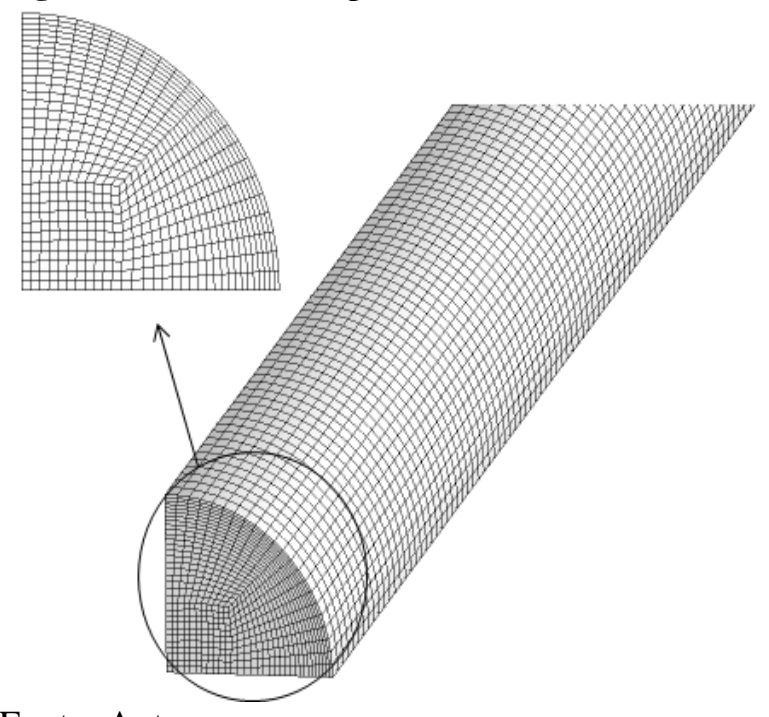

Fonte: Autor.
Esquemas de alta ordem tais como high resolution e second order backward Euler disponíveis no software foram utilizados na discretização dos termos de advecção e transiente respetivamente. Entretanto, o passo de tempo foi estimado garantindo um Courant number inferior 1 . Foi constatado através do monitoramento de diversas variáveis de interesse que um tempo de 5 s era suficiente para a simulação atingir um estado estacionário. Por outro lado, foi estabelecido um erro residual RMS inferior a $10^{-4}$ para todas as equações de conservação como critério de convergência, sendo comumente utilizado em simulações em escala industrial e sugerido pelo manual do usuário do software.

\section{RESULTADOS E DISCUSSÕES}

Os resultados apresentados nessa seção foram obtidos após a simulação atingir um estado estável. Observe-se na Figura 5a a distribuição do catalisador ao longo do riser. Percebe-se uma região densa no fundo do reator e, diluindo-se conforme o catalisador ascende ao longo do reator. É possível observar após altura de $15 \mathrm{~m}$ uma distribuição mais homogênea do catalisador.

Por outro lado, na região de maior concentração de catalisador existe uma maior troca de calor como mostra a Figura 5 b atingindo temperaturas de $850 \mathrm{~K}$ na fase gás. Devido que as reações de craqueamento catalítico são altamente endotérmicas é possível perceber na Figura $5 b$ que temperatura da fase gasosa diminui conforme regem os diversos componentes da mistura reativa ao longo do reator atingindo uma temperatura de $809 \mathrm{~K}$ na saída do reator.

Note-se na Figura 6 que os pseudocomponentes mais pesados L8 e L9 reagem formando produtos de menor peso molecular. Os pseudo-componentes L6 e L7 atingem rapidamente um máximo de produção à altura de $10 \mathrm{~m}$ aproximadamente, no entanto sua produção decresce devido a reações 
secundarias ou overcraking reportada na literatura também por outros autores (GAO et al., 1999; CHANG et al., 2012). Já o pseudocomponente L5 mostra uma produção máxima na altura de $20 \mathrm{~m}$ permanecendo quase constante no resto do percurso do riser. Os demais pseudo-componentes, todos apresentam um incremento gradual conforme aumenta a altura do reator.

Figura 5 - Perfis de concentração de solido e temperatura do gás

Catalyst. Volume Fraction
-0.024
-0.014
-0.010
0.005
0.000

A)

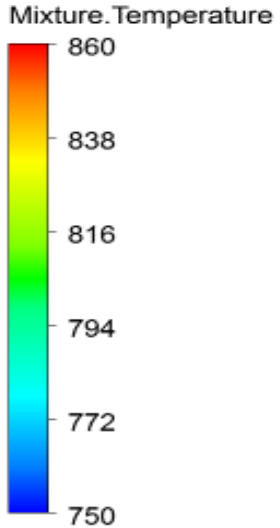

[K]
Figura 6 - Rendimento dos pseudocomponentes

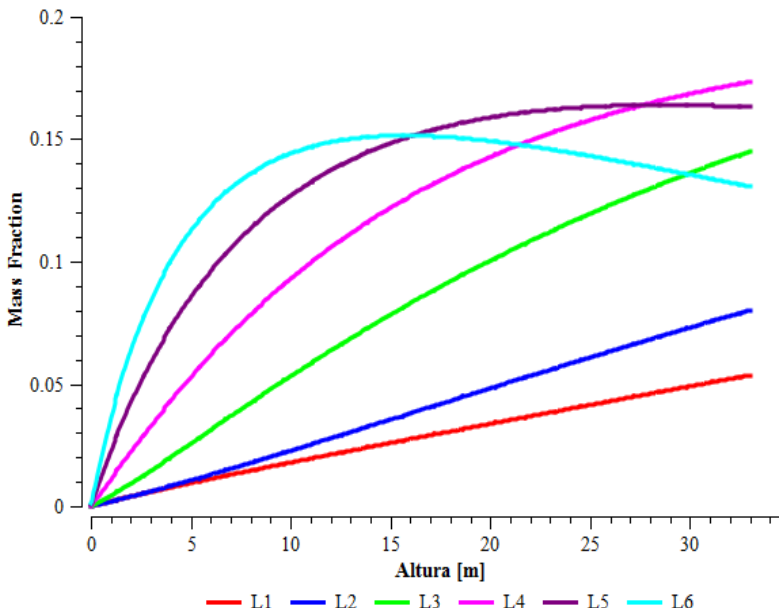

$-\mathrm{L} 1-\mathrm{L} 2-\mathrm{L} 3-\mathrm{L} 4-\mathrm{L} 5-\mathrm{L} 6$

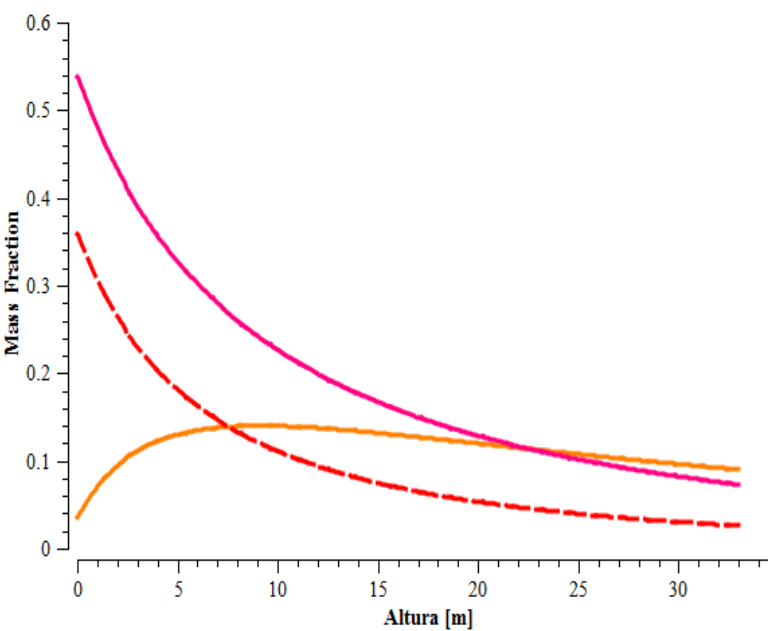

- $\mathrm{L} 7$ - $\mathrm{L} 8--\mathrm{L} 9$
Fonte: Autor.

Entretanto, note-se um aumento de cerca de três vezes da velocidade de entrada da mistura gás na Figura 7a. Cabe salientar que devido á força de arraste, o aumento da velocidade favorece uma maior fluidização da fase sólida dentro do reator.

Sendo o gasóleo uma mistura contínua a divididas em moléculas menores, tem-se uma expansão volumétrica da fase gás e consequentemente um acréscimo da velocidade desta. Na Figura $7 \mathrm{~b}$ pode-se verificar expansão do gás através da diminuição da densidade. Percebe-se que a partir de $20 \mathrm{~m}$ a densidade da fase gás apresenta uma menor variação ao longo do riser conforme as taxas de reação dos compostos mais pesados decrescem. distribuição dos pseudo-componentes tanto na carga de alimentação do reator quanto na saída após a reação pode ser visto na Figura 8. Percebe-se a preferencia do craqueamento dos componentes mais pesados para a produção dos pseudo-componentes associados na faixa da gasolina (L4+L5) característico dos processos FCC. 
Figura 7 - Perfis da velocidade e densidade do gás

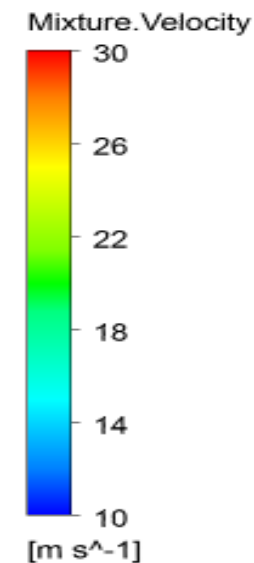

A)

B)

Fonte: Autor.

A fim de validar a predição do modelo, foram comparados os resultados de rendimento dos diversos produtos na saída do riser com dados de planta reportados na literatura, apresentados na Tabela 2. A pesar de algumas diferenças na escala e condições de operação entre o modelo CFD e o riser industrial reportado, pode-se observar uma concordância qualitativa dos resultados, mostrando que o modelo cinético de lumping contínuo pode ser adequado para descrever as reações químicas que ocorrem nos processos FCC.

O modelo apresenta uma boa estimativa da gasolina e LPG com desvios relativos de $1,8 \%$ e $8,3 \%$ respectivamente, entretanto os outros pseudo-componentes apresentaram desvios maiores a 10\%. Essas diferenças podem ser possíveis devido às simplificações feitas na modelagem CFD, bem como a falta de informação das características da carga e catalisador raramente disponível na literatura.
Figura 8 - Distribuição de pseudocomponentes.

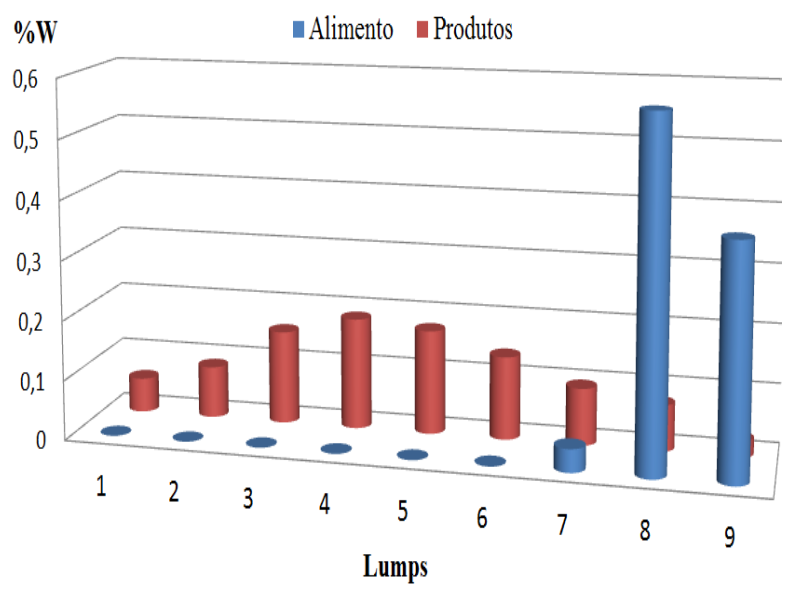

Fonte: Autor.

Tabela 2 - Comparação dos resultados do modelo com dados experimentais.

\begin{tabular}{lccc}
\hline \multicolumn{1}{c}{ Item } & Lump & Modelo & *Chang \\
\hline Coque & L1 & 5,78 & 8,26 \\
Gás seco & L2 & 8,66 & 4,75 \\
LPG & L3 & 15,61 & 15,33 \\
Gasolina & L4-L5 & 36,04 & 39,29 \\
Diesel & L6-L7 & 23,42 & 27,45 \\
Carga não & L8-L9 & 10,48 & 4,92 \\
convertida & & & \\
\hline
\end{tabular}

Fonte: *Chang et al. (2012).

Cabe salientar que existem ainda muitos desafios na incorporação deste tipo de modelos cinéticos na modelagem dos processos FCC via CFD, que envolvem diversos aspectos das reações, tais como a desativação do catalisador, a cinética heterogênea das reações catalíticas, distribuição assimétrica do catalisador, etc., que precisam ser estudados amplamente no futuro.

\section{CONCLUSÕES}

Os resultados mostraram a não uniformidade do escoamento gás-solido no reator $\mathrm{CFB}$, ressaltando que os efeitos hidrodinâmicos sobre a temperatura de reação, tempo de residência e distribuição do 
catalisador são importantes sobre a cinética das reações FCC.

Por outro lado, observou-se nos resultados que a abordagem cinética de lumping contínuo, em que carga foi considerada sendo uma mistura continua de pseudo-componentes, permitiu estimar de forma adequada o rendimento dos produtos de uma unidade FCC industrial.

Finalmente, a modelagem CFD em junção com a abordagem cinética de lumping continua torna-se uma ferramenta útil e inovadora para descrever misturas reativas bastante complexas; sendo apenas necessário um numero reduzido de parâmetros cinéticos que podem ser estimados com certa facilidade na maioria das refinarias.

\section{NOMENCLATURA}

$g \quad$ Fase gás

$s \quad$ Fase particulada

$i \quad$ Espécie ou lump

$\varepsilon \quad$ Fração volumétrica

$\rho \quad$ Densidade $\left[\mathrm{kg} / \mathrm{m}^{3}\right]$

$v \quad$ Velocidade $[\mathrm{m} / \mathrm{s}]$

$k_{i} \quad$ Reatividade da espécie $i[1 / \mathrm{s}]$

$\theta \quad$ Propriedade especifica do lump

$\alpha \quad$ Coeficiente exponencial

$C_{i} \quad$ Concentração da espécie $i$ [\% $\%$

$t \quad$ Tempo [s]

$\tau$ Tensor esforço $\left[\mathrm{N} / \mathrm{m}^{2}\right]$

$\vec{g} \quad$ Gravidade $\left[\mathrm{m} / \mathrm{s}^{2}\right]$

$p \quad$ Pressão $[\mathrm{Pa}]$

$T \quad$ Temperatura [K]

$h \quad$ Entalpia térmica $[\mathrm{J} / \mathrm{Kg}]$

$\beta \quad$ Coeficiente de arraste $\left[\mathrm{kg} / \mathrm{m}^{3}\right.$.s $]$

$Q_{r} \quad$ Calor de reação $\left[\mathrm{J} / \mathrm{m}^{3} \mathrm{~s}\right]$

$Q_{g / s} \quad$ Troca de calor interfacial [ $\left.\mathrm{J} / \mathrm{m}^{3} . \mathrm{s}\right]$

$Y_{g}^{i} \quad$ Fração mássica da espécie $\mathrm{i}$

$\lambda$ Condutividade térmica $[(\mathrm{J} / \mathrm{s}) / \mathrm{K} . \mathrm{m}]$

$\Gamma_{i} \quad$ Difusividade da espécie $i\left[\mathrm{~m}^{2} / \mathrm{s}\right]$

$\phi \quad$ Coeficiente de atividade do catalisador
$R_{i} \quad$ Taxa liquida de reação da espécie $i$ $\left[\mathrm{kg} / \mathrm{m}^{3} \mathrm{~s}\right]$

\section{REFERÊNCIAS}

ADAM, M., CALEMMA, V., GALIMBERTI, F., GAMBARO, C., HEISZWOLF, J., OCONE, R. Continuum lumping kinetics of complex reactive system. Chemical Engineering Science, v76, p.154164. 2012.

ANCHEYTA, J. Modeling and Simulations of catalytic reactors for petroleum refining. New Jersey: John Wiley \& Sons, 2011.

BASAK, K., SAU, M., MANNA, U., VERMA R. P. Industrial hydrocracker model based on novel continuum lumping approach for optimization in petroleum refinery. Catalysis Today, v98, p.253-264. 2004.

BRASIL, N., ARAUJO, M., SOUSA, E., QUELHAS, A., PASSOS, C., LAGE, D., ABADIE, E., CORDEIRO, F., KRAHL, I., FARAH, M., CAMARGO P., PINTO, R. Processamento de Petróleo e Gás: petróleo e seus derivados, processamento primário, processos de refino, petroquímica, meio ambiente. Rio de Janeiro: LTC, 2012.

CHANG, J., MENG, F., WANG, L., ZHANG, K., CHEN, H., YANG, Y. CFD investigation of hydrodynamics, heat transfer and cracking reaction in a heavy oil riser with bottom airlift loop mixer. Chemical Engineering Science, v78, p.128-143. 2012.

ELIZALDE, I., ANCHEYTA J. On the detailed solution and application of the continuous kinetic lumping modeling to hydrocracking of heavy oils. FUEL Journal, v90, p.3542-3550. 2011.

FAHIM, M. A., ALSAHHAF T. A., ELKILANI, A. Fluidized Catalytic 
Cracking. Fundamentals of Petroleum

Refining. Amsterdam: Elsevier, 2010.

GIDASPOW, D. Multiphase flow and Fluidization: Continuum and Kinetic Theory Descriptions. Boston: Academic Press, 1994.

GRAY, J. H., HANDWERK, G. E. Petroleum Refining Technology and Economics. New York: Marcel Dekker, 2001.

GAO, J., XU, C., LIN, S., YANG, G., GUO, Y. Advance model for turbulent gas-solid flow and reaction in FCC riser reactors. AIChE Journal, v45, p.1095-1113. 1999.

PÄRSSINEN, J. H., ZHU, J. X. Particle velocity and flow development in a long and high-flux circulating fluidized bed riser. Chemical Engineering Science, v56, p.52955303. 2001.

LAXMINARASIMHAN, C. S., VERMA, R. P., RAMACHANDRAN, P. A. Continuous lumping model for simulation of hydrocracking. AIChE Journal, v42, p.26452653. 1996.

LI, J., LUO, Z. H., LAN, X. Y., XU, C. M., GAO, J. S. Numerical Simulation of the turbulent gas-solid flow and reaction in polydisperse FCC riser reactor. Powder Technology, v237, p.564-580. 2013.

SADEGHBIGI, R. Fluid Catalytic Cracking Handbook. Houston: ButterworthHeinemann, 2000.

WILCOX, D. C. Turbulence Modelling for CFD. California: Griffin, 1993.

\section{AGRADECIMENTOS}

Os autores gostariam de agradecer a Petrobrás pelo apoio financeiro, bem como ao departamento de engenharia química da Heriot-Watt University em Edinburgh, UK pela parceria e as contribuições acadêmicas neste trabalho. 